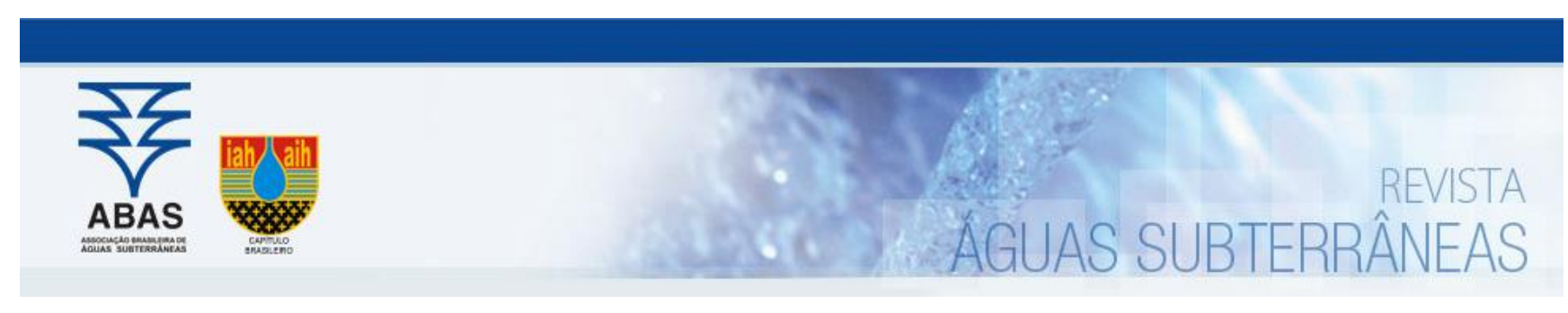

Artigos

\title{
Simulação matemática do transporte de óleo mineral isolante na zona vadosa considerando a constante dielétrica e o índice de plasticidade
}

\section{Mathematical simulation of the transport of isolanting mineral oil in the vadose zone considering the dielectric constant and the plasticity index}

\author{
Muriel Edyth Lumsden Szymanski Patricio; Marcio Roberto Schneider1; Konrad Ziemowit Miotliński; Admir José Giachini1; \\ Alzete Martins Quadros ${ }^{\bowtie}$
}

1 Universidade Federal de Santa Catarina (UFSC), Florianópolis, SC

2 ELETROSUL, Florianópolis, SC
\uriel.edyth@gmail.com, mrs2007@hotmail.com, konrad.miotlinski@ufsc.br, admir.giachini@gmail.com, alzete@eletro- sul.gov.br

\author{
Palavras-chave: \\ Óleo mineral isolante. \\ Constante dielétrica. \\ LNAPL. \\ Fluxo. \\ Contaminação do solo.
}

\begin{abstract}
Resumo
Acidentes ou falhas em equipamentos elétricos em subestações de energia podem resultar em vazamentos de óleo mineral isolante (OMI), ocasionando contaminação do solo e da água subterrânea. A modelagem matemática é uma importante ferramenta para a previsão dos impactos destes vazamentos e para a determinação do tempo de resposta das ações emergenciais. Um dos simuladores mais utilizados neste processo é o Hydrocarbon Spill Screening Model (HSSM), distribuído pela U.S.EPA. Contudo, esse e outros modelos baseados na equação de Nutting (1934), não consideram o efeito da constante dielétrica na determinação da mobilidade dos contaminantes. 0 objetivo deste estudo contemplou a avaliação da influência da constante dielétrica na modelagem matemática de OMI e analisou a simulação de derramamento de OMI em cinco subestações de energia elétrica com o simulador Hydrocarbon Spill Screening Model (HSSM). Os resultados indicaram que em solos constituídos de argilas expansivas, a inclusão da constante dielétrica na modelagem matemática do transporte na zona vadosa pode representar um acréscimo de até três ordens de grandeza à condutividade hidráulica ao OMI, influenciando as ações de contingenciamento ambiental ponderadas sobre essas estimativas.

Abstract

Accidents and failures of electrical equipment at power substations may result in infiltration of insulating mineral oil (OMI) into the soil leading to contamination. Mathematical modeling is an important tool to predict the impacts and to determine the response time of emergency actions. One of the most used software for this purpose is the Hydrocarbon Spill Screening Model (HSSM), distributed by the U.S. EPA. It is based on the equation of Nutting (1934) and it is capable of predicting $\mathrm{OMI}$ traveling in the vadose zone, but it does not consider (as for other models) the effect of the dielectric constant in determining the mobility of contaminants. Hence, the objective of the present study included an evaluation of the influence of the dielectric constant in the mathematical modeling of OMI and analyzed OMI spill simulations in five electric power substations with the Hydrocarbon Spill Screening Model (HSSM). The results indicated that, in expansive clay soils, the inclusion of the dielectric constant may represent an increase of up to three orders of magnitude in the hydraulic conductivity of the $\mathrm{OMI}$, which influences contingency plans needed in case of spills.
\end{abstract}

Keywords:

Transformer oil.

Dieletric constant.

LNAPL.

Flow.

Soil pollution.

Revisão por pares.

Recebido em: 21/02/2019.

Aprovado em: 13/10/2020.
DOI: http:/dx.doi.org/10.14295/ras.v34i3.29501

\section{INTRODUÇÃO}

Empresas do setor elétrico enfrentam problemas ambientais frequentes devido à ocorrência de falhas que resultam em vazamentos de óleos minerais isolantes (OMI), utilizados para o isolamento elétrico e refrigerante em transformadores de potência (MÓDENES et al., 2018). Por serem imiscíveis e menos densos que a água, quando liberados acidentalmente no ambiente, estes fluídos dielétricos recebem a denominação de fase livre (CONAMA, 2009), em inglês light non aqueousphase liquid (LNAPL) (API, 2018). A primeira linha de defesa para o enfrentamento do problema da contaminação de ambientes terrestres ou aquáticos por vazamentos de OMI é a prevenção, conforme diretrizes da ANEEL (2015). Neste contexto, a modelagem matemática vem sendo adotada como instrumento para auxiliar na previsão e planejamento de ações preventivas e de contingenciamento para os casos de vazamentos de óleo em diversos tipos de empreendimentos 
Os fenômenos envolvidos na modelagem matemática do transporte do LNAPL na zona não saturada são complexos, influenciados por diversos parâmetros importantes, a exemplo das propriedades da água, ar e LNAPL (densidade, viscosidade e tensão superficial) e propriedades do solo (condutividade hidráulica, porosidade e a fração residual dos fluídos no meio poroso) (BEAR; CHANG, 2010). Os simuladores de transporte de LNAPL na zona não saturada disponíveis no mercado consideram a base conceitual estabelecida por Nutting (1931), que utiliza a relação entre densidade $\left(\rho\left[\mathrm{Kg} / \mathrm{m}^{3}\right]\right)$ e viscosidade ( $\mu$ [Pa.s]) no cálculo da mobilidade de fluidos polares e apolares no meio poroso, negligenciando os efeitos das interações elétricas entre o solo e o fluído. Estudos realizados por Budhu et al. (1991), Oliveira (2001), Amorim Júnior (2007), Cardoso (2011), Machado et. al. (2016) e Patricio (2019) demonstraram que a mobilidade dos fluidos em solos argilosos pode ser influenciada pela interação elétrica entre os argilominerais presentes em solos expansivos (ex.: montmorilonita e vermiculita) e os fluídos polares como a água, dependendo da constante dielétrica $(\varepsilon)$. Os autores concluíram que, em relação à água $(\varepsilon=80)$, as condutividades hidráulicas ao LNAPL na zona não saturada podem ser superior a três ordens de magnitude quando o LNAPL é um composto apolar, como no caso do óleo diesel $(\varepsilon=2,13)$, do tetracloreto de carbono $(\varepsilon=2,20)$ e da gasolina pura $(\varepsilon=2,07)$

Os estudos realizados por Cardoso (2011), Machado et al. (2016) e Corseuil et al. (2020) demonstraram que, a partir do índice de plasticidade (IP) do solo, é possível obter uma estimativa sobre a superfície específica do argilomineral e da capacidade de troca catiônica do solo. Logo, a presença de argilominerais expansivos está diretamente relacionada ao IP, portanto, quanto maior o índice, maior a superfície específica e mais expansivo é o solo argiloso (CORSEUIL et al., 2020). A partir desta premissa, Machado et al. (2016) desenvolveram uma formulação matemática capaz de determinar a condutividade hidráulica ao LNAPL, relacionando a influência da constante dielétrica e o índice de plasticidade do solo. Neste contexto, as subestações de energia elétrica são exemplos de empreendimentos onde a interação elétrica entre o solo e o fluído deve ser considerada nas simulações do transporte do LNAPL, visto que grande parte das subestações são construídas sobre solos argilosos.

O objetivo deste estudo foi comparar o emprego da metodologia definida por Nutting (1934) com a metodologia proposta por Machado et al. (2016), que considera a influência da constante dielétrica e do índice de plasticidade, no transporte do LNAPL em solos argilosos expansivos. Além disso, foram avaliadas as principais implicações dessas considerações em cinco subestações de energia elétrica, na elaboração de planos de ações emergenciais, a partir da simulação de cenários de derramamento de óleo mineral isolante com o simulador Hydrocarbon Spill Screening Model (HSSM) (CHARBENEAU; WEAVER; LIEN, 1995).

\section{MATERIAL E MÉTODOS}

\subsection{Modelo matemático}

O modelo matemático empregado nas simulações foi o Hydrocarbon Spill Screening Model (HSSM) (CHARBENEAU; WEAVER; LIEN, 1995), distribuído pela U.S.EPA. O modelo simula o fenômeno de infiltração de substâncias imisciveis em água e a redistribuição do fluído na zona vadosa, utilizando como base a formulação de Green - Ampt (GREEN; AMPT, 1911; Eq. 1) e a teoria da onda cinética, como uma alternativa às formulações numéricas propostas por Darcy-Buckingham (1907) e Richards (1928) (LIBARDI, 2005). O HSSM assume a premissa que o movimento é unidimensional, o que caracteriza bons resultados quando o escoamento de LNAPL ocorre em grandes áreas, uma vez que se pode negligenciar o escoamento lateral (WEAVER; CHARBENEAU; LIEN, 1994 e CHARBENEAU; WEAVER; LIEN, 1995):

$$
t_{0}=\eta \frac{S_{o}}{K_{e o}}\left[z_{f}-H \ln \left(\frac{z_{f}+H}{H}\right)\right]
$$

onde $K_{e o}$ é a condutividade efetiva ao LNAPL [m/s], $\eta$ é a porosidade total [adimensional], $S_{o}$ é a saturação do óleo [adimensional], $H$ é a carga de LNAPL na frente de migração [m], $z_{f}$ é a posição do fluído [m] e $t$ é o tempo [s].

A condutividade efetiva ao LNAPL $\left(K_{e o}[\mathrm{~m} / \mathrm{s}]\right)$ é determinada por meio do produto entre a condutividade hidráulica ao LNAPL $\left(K_{s o}[\mathrm{~m} / \mathrm{s}]\right)$ e a permeabilidade relativa ao LNAPL ( $k_{\text {ro }}$ [adimensional]), como mostra a Equação 2:

$$
K_{e o}=K_{\text {so }} k_{\text {ro }}
$$

A permeabilidade relativa ao LNAPL $\left(k_{r o}\right)$ é determinada por Brooks e Corey (1964) (Eq. 3). Já para o cálculo da condutividade hidráulica ao LNAPL $\left(K_{s o}[\mathrm{~m} / \mathrm{s}]\right)$ na zona vadosa o HSSM (CHARBENEAU; WEAVER; LIEN, 1995) considera a formulação matemática de Nutting (1934) (Eq. 4):

$$
k_{r o}=\left(\frac{S_{o}-S_{o r}}{1-S_{o r}}\right)^{2}\left[\left(\frac{S_{o}+S_{w}-S_{w r}}{1-S_{w r}}\right)^{\xi-2}-\left(\frac{S_{w}-S_{w r}}{1-S_{w r}}\right)^{\xi-2}\right]
$$

$$
K_{s o}=k \frac{\rho_{i}}{\mu_{i}}
$$

onde $k\left[\mathrm{~m}^{2}\right]$ é a permeabilidade intrínseca e $\rho_{i}\left[\mathrm{Kg} / \mathrm{m}^{3}\right]$ e $\mu_{i}$ [Pa.s] são a densidade e viscosidade do fluído, respectivamente.

No entanto, para avaliar a influência da constante dielétrica do fluído e do índice de plasticidade do solo é utilizada a equação de Machado et al. (2016) (Eq. 5): 
$K_{S O}=K_{S W} \frac{\rho_{o}}{\rho_{w}} \frac{\mu_{w}}{\mu_{o}} 10^{a\left(\frac{\varepsilon_{W}}{\varepsilon_{o}}-1\right)^{b}\left[1-\exp \left(\frac{-c I P}{c+I P} \log \left(\frac{k_{r e f}}{K_{S W}}\right)\right)+\frac{I P}{c+I P}\left(d \log \left(\frac{k_{r e f}}{K_{S W}}\right)+\frac{e}{\left(1-S_{W}\right)}\right)\right]}$

onde $K_{s w}$ é a condutividade hidráulica à água $[\mathrm{m} / \mathrm{s}], \rho_{o}$ é a densidade do fluído $\left[\mathrm{kg} / \mathrm{m}^{3}\right], \rho_{w}$ é a densidade da água [kg/m³], $\mu_{o}$ é a viscosidade do fluído [Pa.s], $\mu_{w}$ é a viscosidade da água [Pa.s], $\varepsilon_{o}$ a constante dielétrica do fluído [adimensional], $\varepsilon_{w}$ a constante dielétrica da água [adimensional] e IP o índice de plasticidade [adimensional]. Já os parâmetros a, b, c, d s e $k_{\text {ref }}$ são constantes da equação, equivalendo a

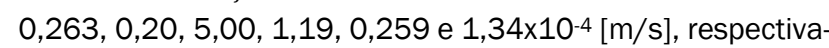
mente.

\subsection{Modelo conceitual}

O modelo conceitual definido para o modelamento matemá- tico do derramamento de óleo isolante em cinco subestações de energia elétrica é representado pela Figura 1 e inclui as seguintes condições de contorno: (a) o derramamento foi de 2,5 $\mathrm{m}^{3}$ de óleo mineral isolante na superfície do solo seco; (b) a espessura inicial da lâmina de óleo foi de 0,1 m sobre a superfície do terreno; (c) a área contaminada é equivalente à $250 \mathrm{~m}^{2}$; (d) a lâmina de óleo permanece constante por 10 segundos e após esse tempo a altura da lâmina de óleo inicia o decaimento; e (e) a posição do lençol freático é de 5 metros abaixo da superfície do terreno.

Figura 1. -Modelo conceitual utilizado para a simulação no HSSM

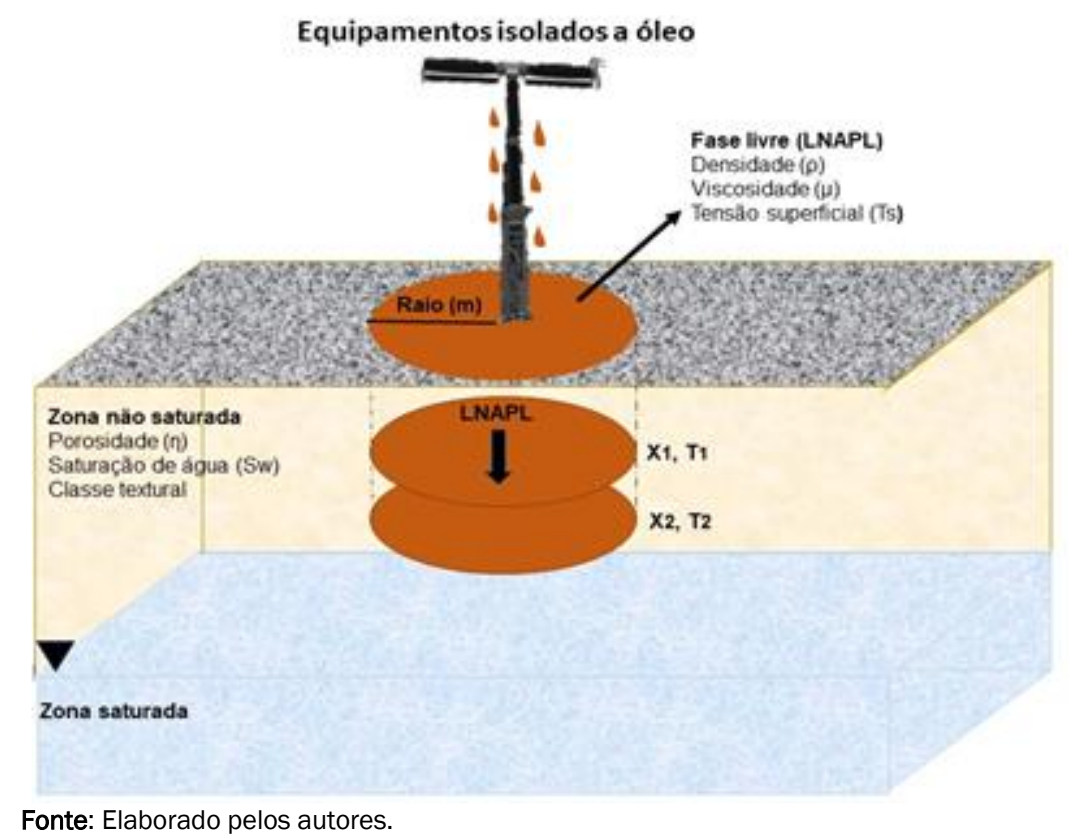

\section{3. Áreas de estudo}

Foram selecionadas cinco subestações de energia elétrica situadas na região sul do Brasil (localização não divulgada por restrição contratual), sendo que para cada área foram realizadas as seguintes análises: textura do solo, índice de plasticidade (IP), densidade aparente $\left(\rho_{a}\left[\mathrm{Kg} / \mathrm{m}^{3}\right]\right)$, densidade da partícula $\left(\rho_{p}\left[\mathrm{Kg} / \mathrm{m}^{3}\right]\right)$ e ensaio da condutividade hidráulica à água $\left(K_{\mathrm{sw}}[\mathrm{m} / \mathrm{s}]\right)$ na zona vadosa. Os quatro primeiros parâmetros foram analisados em laboratório, sendo que a definição da textura do solo, densidade aparente do solo e da densidade de partículas foram analisadas conforme estabelecido pela EMBRAPA (1997). O índice de plasticidade foi determinado por meio da metodologia estabelecida pela ABNT NBR 7180:2016 (2016). O ensaio de condutividade hidráulica à água foi executado em campo (in situ) com o emprego de um Permeâmetro Guelph (Figura 2). No processo de coleta das amostras de solo a camada superficial de brita em cada subestação de energia elétrica foi inicialmente removida. 0 ensaio de condutividade hidráulica na zona não saturada foi realizado em condições de clima seco e estável, geralmente após três dias sem chuva. Esse cuidado é necessário, principalmente para a determinação da condutividade hidráulica à água na zona vadosa, uma vez que o solo, logo após períodos de chuva, encontra-se saturado, impossibilitando a realização do estudo.

A partir dessas coletas e ensaios foi possível obter informações sobre a porosidade $(\eta)$, saturação de água $\left(S_{w}\right)$ e saturação residual de água $\left(S_{w r}\right)$. A porosidade $(\eta)$ foi determinada por meio da equação 6 , enquanto a saturação de água $\left(S_{w}\right)$ e a saturação residual de água $\left(S_{w r},\right)$ na zona vadosa foram determinadas a partir dos parâmetros de van Genutchen $\left(\theta_{r}\right.$ e $\left.\theta_{s}\right)$, identificadas pelas equações 7 e 8 (HEATH, 1983 e KAR- 
POUZAS et al., 2006). Os valores de Van Genutchen foram obtidos a partir do estudo realizado por Tomasella e Hodnett (2002), considerando uma pressão capilar de $-1500 \mathrm{kPa}$, também conhecido como ponto de murcha. Em relação à saturação do óleo $\left(S_{\text {or }}\right)$, foi considerado o valor 0,1, de acordo com o estudo realizado por Mercer e Cohen (1990).

$$
\begin{aligned}
& \rho_{a}=\rho_{p}(1-\eta) \\
& S_{w}=\frac{\theta_{i}}{\eta} \\
& S_{w r}=\frac{\theta_{r}}{\theta_{s}}
\end{aligned}
$$

Figura 2 - Coletas de amostras de solo em campo para as análises de densidade aparente, granulometria e índice de plasticidade (a e b). Ensaio com o Permeâmetro Guelph (c). Legenda: a) Anel volumétrico para a coleta de solo. b) Amostra de solo deformada, coletada para a análise granulométrica. c) Ensaio com o permeâmetro Guelph

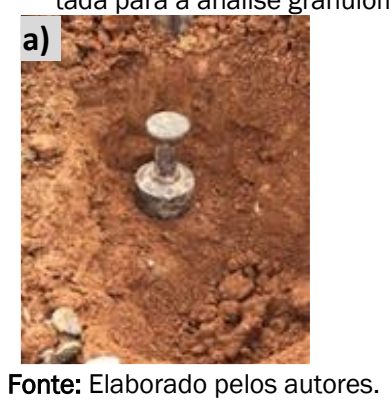

\subsection{Dados de entrada para as simulações}

A definição dos dados de entrada das simulações do transporte dos $\mathrm{OMI}$ em cinco subestações de energia elétrica toma-

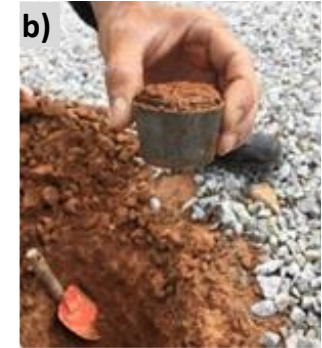

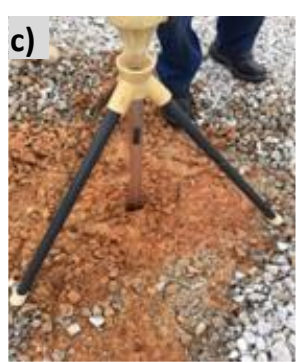

ram como base as formulações matemáticas empregados no simulador HSSM (CHARBENEAU; WEAVER; LIEN, 1995) e as premissas definidas no modelo conceitual, conforme apresentado na Tabela 1.

Tabela 1 - Parâmetros utilizados nas simulações dos cinco estudos de caso

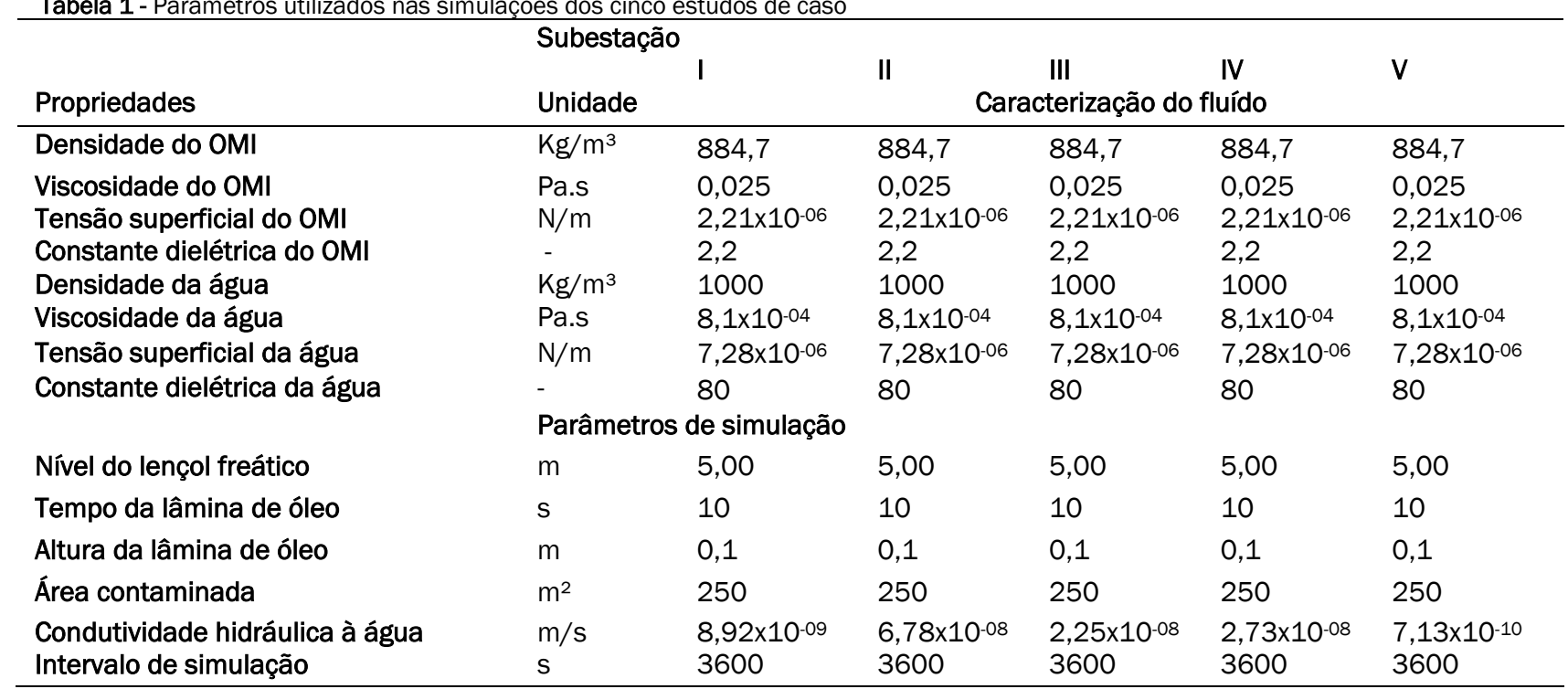

Fonte: ANP (2007); Oliveira (2001); Petrobras (2015); Wada (2011).

\section{RESULTADOS E DISCUSSÃO}

\subsection{Caracterização do solo}

Com base nos resultados das análises granulométricas e de acordo com a classificação da EMBRAPA (2006), os solos das cinco subestações foram classificados como de textura argilo- sa. Os ensaios com o permeâmetro Guelph evidenciaram que a condutividade hidráulica à água na zona vadosa, nas cinco subestações, variou em duas ordens de grandeza, entre

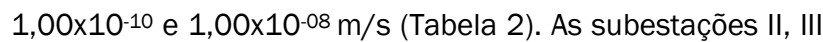
e IV foram aquelas que apresentaram as maiores condutividades hidráulicas à água. 
Tabela 2 - Resultados das análises de solo e da permeabilidade dos solos das cinco subestações

\begin{tabular}{|c|c|c|c|c|c|c|c|c|c|c|c|c|}
\hline \multicolumn{2}{|c|}{$\begin{array}{l}\text { Parâmetros } \\
\text { Unidades }\end{array}$} & $\begin{array}{r}\boldsymbol{\rho}_{a} \\
\mathrm{Kg} / \mathrm{m}^{3}\end{array}$ & $\begin{array}{c}\boldsymbol{\rho}_{p} \\
\mathrm{Kg} / \mathrm{m}^{3}\end{array}$ & $\begin{array}{l}\eta \\
-\end{array}$ & $\begin{array}{l}\text { \%Ar } \\
-\end{array}$ & $\begin{array}{l}\% \mathrm{Si} \\
-\end{array}$ & $\begin{array}{l}\text { \%Ag } \\
-\end{array}$ & 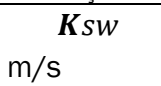 & $S_{w}$ & $S_{w r}$ & $S_{o r}$ & $\begin{array}{l}\text { IP } \\
-\end{array}$ \\
\hline \multirow{5}{*}{ 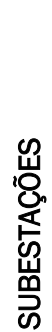 } & I & 1.060 & 2.950 & 0,64 & 7,1 & 29,7 & 63,2 & $8,92 \times 10^{-09}$ & 0,43 & 0,49 & 0,1 & 26,52 \\
\hline & II & 1.340 & 2.580 & 0,42 & 14,6 & 36,3 & 49,1 & $6,78 \times 10^{-08}$ & 0,66 & 0,49 & 0,1 & 21,45 \\
\hline & III & 1.320 & 2.660 & 0,50 & 4,3 & 31,6 & 64,1 & $2,25 \times 10^{-08}$ & 0,55 & 0,49 & 0,1 & 18,00 \\
\hline & IV & 1.510 & 2.660 & 0,43 & 5,1 & 31,0 & 64,0 & $2,73 \times 10^{-08}$ & 0,64 & 0,49 & 0,1 & 35,00 \\
\hline & V & 1.390 & 2.590 & 0,49 & 6,1 & 28,1 & 65,8 & $7,13 \times 10^{-10}$ & 0,56 & 0,49 & 0,1 & 27,00 \\
\hline
\end{tabular}

\%Ar: Fração de Areia; \%Si: Fração de Silte; \%Ag: Fração de Argila.

Fonte: Elaborado pelos autores

A partir da caracterização do solo e das informações apresentadas na Tabela 1 foi possível realizar as comparações entre as metodologias de Nutting (1934) e de Machado et al. (2016) para a determinação da condutividade hidráulica ao LNAPL e as simulações de derramamento de óleo mineral isolante (OMI) no programa HSSM (CHARBENEAU; WEAVER; LIEN, 1995).

3.2 Comparação entre a condutividade hidráulica ao OMI $\left(K_{\text {so }}\right)$ obtida pela Equação de Nutting (1934) e a equação de Machado et al. (2016)

Os resultados de simulação no HSSM (CHARBENEAU; WEAVER; LIEN, 1995) nas cinco subestações de energia indicaram que as condutividades hidráulicas ao $\mathrm{OMI}$, obtidas por meio da Equação de Nutting (Eq.4), variaram entre $1 \times 10^{-11} \mathrm{e}$ $1 \times 10^{-09} \mathrm{~m} / \mathrm{s}$. Estes valores consideram que a mobilidade do LNAPL é função da mobilidade do produto $(\rho / \mu)$ e da perme- abilidade intrínseca $(k)$ decorrente. Já os valores calculados por meio da aplicação da formulação de Machado et al. (Eq. 5) variaram entre $1 \times 10^{-08}$ e $1 \times 10^{-07} \mathrm{~m} / \mathrm{s}$ (Tabela 3). A diferença entre as condutividades hidráulicas dos OMI obtidas pelas formulações matemáticas de Nutting (Eq.4) e Machado et al. (Eq. 5) foi de até três ordens de grandeza. Essa diferença corrobora com os estudos realizados por Oliveira (2001), Amorim (2007) e Cardoso (2010), os quais verificaram que, dependendo da composição mineralógica do solo e da constante dielétrica, a condutividade hidráulica ao fluído apolar pode ser até quatro ordens de magnitude maior do que a condutividade hidráulica de um fluído polar. Portanto, a infiltração de fluídos apolares (ex. OMI, gasolina, diesel, querosene de avião e outros) em solos argilosos com presença de argilominerais expansivos pode ter maior velocidade de migração. Já os fluídos polares (ex.: água) tendem a interagir eletricamente com o solo argiloso, motivo que tem levado ao emprego de solos argilosos na impermeabilização em aterros de resíduos.

Tabela 3 - Condutividade hidráulica à água e ao óleo obtida por meio das equações de Nutting (1934) (saída do HSSM) e Machado et al. (2016)

\begin{tabular}{cccc}
\hline Subestação & $\begin{array}{c}\text { Condutividade hidráulica à água } \\
\boldsymbol{K}_{\boldsymbol{s w}}(\mathrm{m} / \mathrm{s})\end{array}$ & \multicolumn{2}{c}{ Condutividade hidráulica ao OMI $\boldsymbol{K}_{\text {so }}$ (m/s) } \\
& & Nutting (Eq. 4) & Machado (Eq. 5) \\
\hline I & $8,92 \times 10^{-09}$ & $2,89 \times 10^{-10}$ & $1,09 \times 10^{-07}$ \\
II & $6,78 \times 10^{-08}$ & $2,20 \times 10^{-09}$ & $1,81 \times 10^{-07}$ \\
III & $2,25 \times 10^{-08}$ & $7,29 \times 10^{-10}$ & $1,10 \times 10^{-07}$ \\
IV & $2,73 \times 10^{-08}$ & $8,84 \times 10^{-10}$ & $1,60 \times 10^{-07}$ \\
V & $7,13 \times 10^{-10}$ & $2,31 \times 10^{-11}$ & $2,97 \times 10^{-08}$ \\
\hline
\end{tabular}

Fonte: Elaborado pelos autores.

\subsection{Simulação da infiltração}

A partir dos resultados apresentados na Tabela 2 e das condições de contorno e propriedades dos fluídos apresentadas naTabela 1, foi possível simular os cinco cenários de simulação, por meio do modelo HSSM (CHARBENEAU; WEAVER; LIEN, 1995) (Figura 3)

A partir dos resultados obtidos com o HSSM, nota-se que a Subestação II apresentou a maior condutividade hidráulica ao óleo $\left(K_{s o}\right)$, igual a $1 \times 10^{-09} \mathrm{~m} / \mathrm{s}$ (Tabela 3$)$, e a mais baixa saturação de óleo (29\%; Tabela 4), resultando em uma condutividade efetiva elevada (Tabela 4) quando comparada com as demais subestações. Esta condição proporciona que o contaminante migre até uma profundidade maior após o vazamento do fluido apolar. Em contraposição, a Subestação V apresentou a menor condutividade hidráulica ao OMI $\left(K_{s o}\right)$, além de apresentar elevadas saturações residuais de água, determinando uma baixa condutividade efetiva $\left(K_{e o}\right)$ e a menor profundidade de migração do OMI (Figura 3 e Tabela 4) e, consequentemente, uma infiltração mínima na zona vadosa (menor que 0,01 m em 100 dias após a contaminação). Já ao comparar as subestações III e IV, as quais são bastante similares em termos de condutividade hidráulica ao OMI (Tabela 3), nota-se que a Subestação III apresentou uma condutividade efetiva maior do que a Subestação IV devido à saturação de água e 
de OMI (Tabela 3 e Tabela 4). Portanto, o contaminante na Subestação III atingiria uma profundidade maior do que na Subestação IV. Apesar da Subestação I apresentar condutivida- dade efetiva superior a Subestação IV, o contaminante apresentou uma velocidade menor, alcançando menores profundidades devido à saturação do OMI em I ser equivalente a 51\%.

Tabela 4 - Resultados da condutividade efetiva ao OMI e da saturação de OMI (obtidas no programa HSSM) para cada uma das subestações de energia elétrica

\begin{tabular}{lll}
\hline Subestação & $\begin{array}{l}\text { Condutividade efetiva do OMI } \boldsymbol{K}_{\boldsymbol{e o}} \\
(\mathrm{m} / \mathbf{s})\end{array}$ & Saturação de OMI $\boldsymbol{S}_{\boldsymbol{o}}(\%)$ \\
\hline I & $3,83 \times 10^{-11}$ & 52,24 \\
II & $6,36 \times 10^{-11}$ & 29,76 \\
III & $4,91 \times 10^{-11}$ & 40,14 \\
IV & $2,93 \times 10^{-11}$ & 31,13 \\
V & $1,44 \times 10^{-12}$ & 39,01 \\
\hline
\end{tabular}

Fonte: Elaborado pelos autores.

Figura 3 - Tempo da frente de escoamento do óleo mineral isolante (OMI), obtido por meio do HSSM, para cada uma das subestações analisadas, em função de profundidade

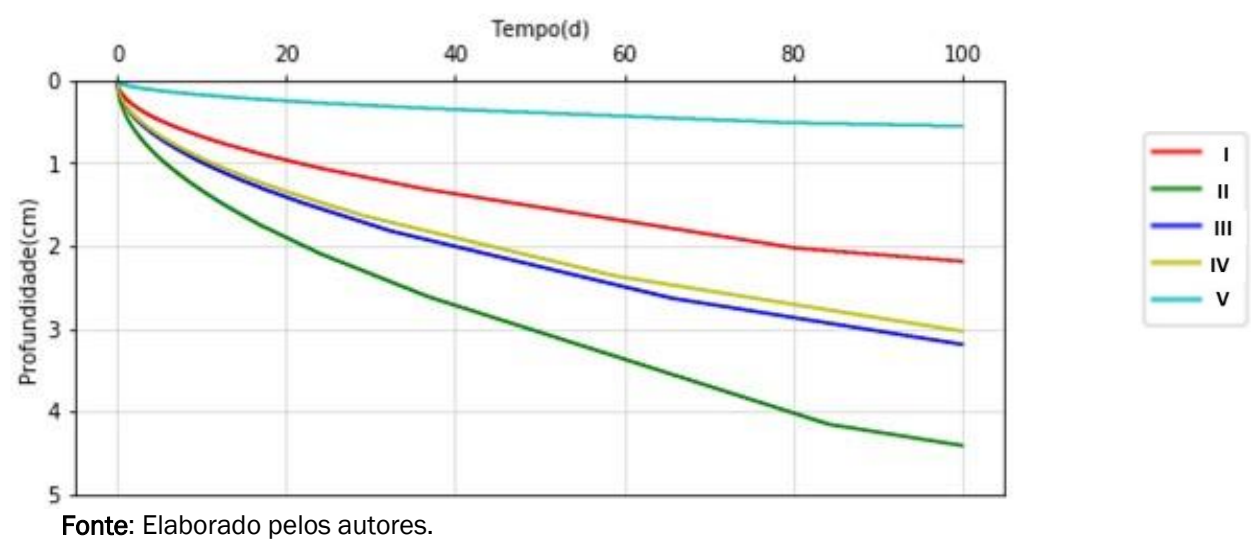

Devido às saturações de OMI, porosidades dos solos das subestações e às baixas condutividades efetivas, a espessura da lâmina infiltrada na zona vadosa também foi reduzida, ocasionando o empoçamento de OMI na superfície do solo (Figura 4). As subestações I e III, que apresentaram as maiores porosidades, foram justamente aquelas que apresentaram a maior espessura de OMI infiltrado. As subestações II e IV apresen- tam porosidades semelhantes (43\%), no entanto a saturação de OMI em IV foi superior, garantindo que uma maior espessura de OMI infiltrasse na zona vadosa da Subestação IV. E por fim, a Subestação $V$, que apesar de possuir uma porosidade equivalente a $49 \%$ (superior às subestações II e IV), apresentou uma espessura da lâmina de OMI infiltrada mínima, provavelmente em decorrência da saturação do OMI.

Figura 4 - Espessura da lâmina de óleo mineral isolante (OMI) infiltrada na zona vadosa durante a migração da frente de escoamento nas subestações analisadas

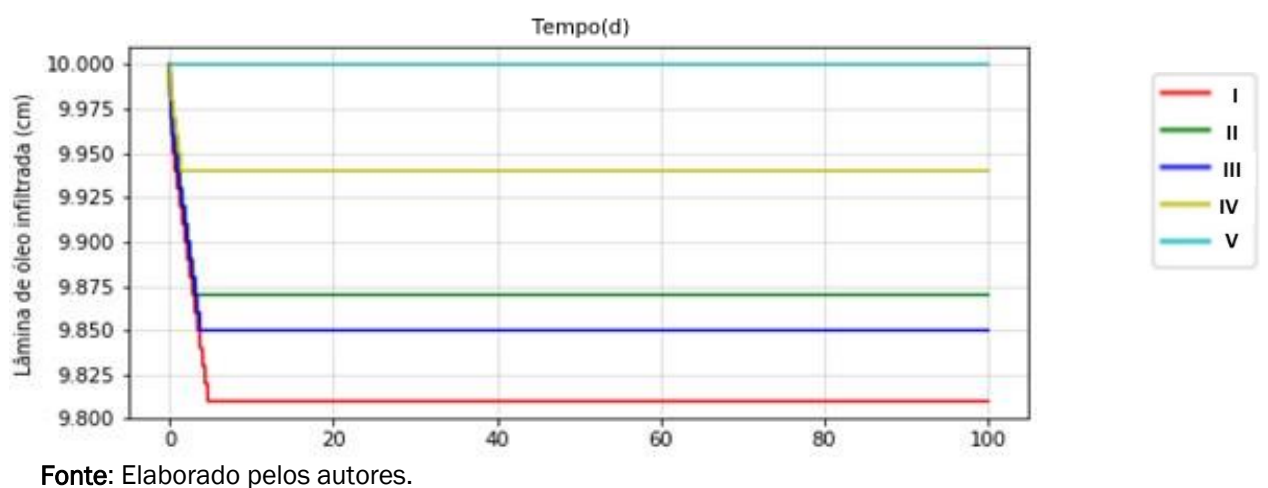

Conforme discutido no item 3.2, o HSSM utiliza a equação de Nutting para determinar a condutividade hidráulica ao óleo na zona não saturada, o que consequentemente influencia diretamente a determinação da condutividade efetiva ao óleo $\left(K_{\mathrm{eo}}\right)$ (Eq. 2) e o tempo da frente de migração do LNAPL (Eq.1)
Desta forma, ao negligenciar a interação entre o fluído e o solo, por meio da relação entre a constante dielétrica do fluído e do índice de plasticidade, a condutividade hidráulica ao óleo pode ser subestimada, resultando em uma migração do LNAPL muito mais lenta do que poderia ser obtida na prática 
e, assim, dificultando a elaboração de ações emergenciais aderentes à realidade.

\section{CONCLUSÕES}

As ferramentas computacionais disponiveis no mercado para a simulação de vazamentos de fluidos orgânicos imisciveis em água (LNAPL) utilizam a formulação matemática de Nutting (1934), na qual a mobilidade do fluido no solo é determinada em função da sua densidade, viscosidade e da permeabilidade intrínseca do meio poroso. Contudo, os resultados deste estudo demonstraram que a constante dielétrica de substâncias apolares, como o óleo mineral isolante (OMI), também podem influenciar a migração vertical desses fluidos na zona vadosa de solos argilosos. As simulações do transporte de OMI em cinco subestações de energia elétrica demonstraram que o valores das condutividades hidráulicas para os OMI foram até três ordens de grandeza maiores quando a constante dielétrica foi considerada, em comparação com o emprego do modelo de Nutting (1934). Estes resultados corroboram com a teoria de que os argilominerais expansivos do solo não interagem eletricamente com os fluídos apolares (baixa constante dielétrica), a exemplo do OMI. De forma contrária, a água, como substância polar $(\varepsilon=80)$, apresentou forte interação com o solo argiloso, refletindo na sua retenção no meio poroso.

Apesar do HSSM ser um simulador amplamente difundido e testado mundialmente, as validações efetuadas pelos seus desenvolvedores foram apenas realizadas com ensaios de permeabilidade em colunas preenchidas com areia e com o fluido Soltrol 220, um solvente orgânico. Wada (2011) também realizou a validação do HSSM por meio de ensaios de infiltração em colunas de areia, mas utilizando OMI. Como era esperado, todos os autores obtiveram bons resultados de calibração com o modelo físico. Contudo, nenhum dos estudos contemplou a migração de líquidos orgânicos em meio poroso argiloso, o que deixa uma lacuna sobre a validade do HSSM na aplicação em solos argilosos.

No desenvolvimento do presente estudo foi verificado que muitas subestações de energia elétrica foram construídas sobre embasamento de material argiloso natural ou aterro compactado. Desta forma, é de fundamental importância que os procedimentos de modelagem matemática de vazamentos de OMI levem em consideração a influência da constante dielétrica sobre a migração vertical dos fluidos apolares, sob pena de subestimar os impactos do escoamento dos fluídos apolares nos solos argilosos. Essa recomendação também pode ser estendida aos empreendimentos industriais ou comerciais que armazenam ou transportam substâncias químicas apolares e que, no contexto do planejamento de ações preventivas e de contingenciamento ambiental, se utilizem de modelos matemáticos de transporte de LNAPL.

\section{REFERÊNCIAS}

ASSOCIAÇÃO BRASILEIRA DE NORMAS TÉCNICAS. NBR 7180 -
Solo- Determinação do limite de plasticidade. Rio de Janeiro: ABNT, 2016.

AGÊNCIA NACIONAL DE ENERGIA ELÉTRICA. Resolução normativa $n^{\circ}$ 669: Regulamenta os Requisitos Mínimos de Manutenção e o monitoramento da manutenção de instalações de transmissão de Rede Básica. Brasilia, 2015. Disponível em: $\quad$ http://www2.aneel.gov.br/aplicacoes/audiencia/arquivo/2014/022/resultado/ren2015669.pdf. Acesso em: 08 out. 2020.

AMORIM JÚNIOR, C. J. Avaliação dos critérios de impermeabilização de bacias de contenção da norme ABNT NBR 17505-2/2006 para terminais de armazenamento de petróleo e derivados. 127 f. 2007. Dissertação (Mestrado) Universidade federal de Santa Catarina, 2007.

ANP, AGÊNCIA NACIONA DO PETRÓLEO. Resolução ANP no 36, de 13.11.2007, 2007.

API, American Petroleum Institute (org.). Managing risk at LNAPL sites: Frequently asked questions. Washington: American Petroleum Institute, 2018.

BEAR, Jacob; CHENG, Alexander H.-D. Modeling Groundwater Flow and Contaminant Transp: theory and applications of transport in porous media. 23. ed. New York: Springer, 2010. 850 p. https://doi.org/10.1007/978-1-4020-6682-5

BROOKS, R. H.; COREY, A T. Hydraulic properties of porous media. Hydrology Papers, Colorado State University. Fort Collins CO, v. 3, n. 3, p. 27 p. 1964.

BUDHU, M.; GIESE JR., R. F.; CAMPBELL, G.; BAUMGRASS, L. The permeability of soils with organic fluids. Canadian Geotechnical Journal, v. 28, n. 1, p. 140-147, 1991. https://doi.org/10.1139/t91-015

CARDOSO, L. DA SILVA PAES. Estudo do transporte de poluentes imiscíveis em solo. 152 f. 2001. Dissertação (Mestrado)- Universidade Federal da Bahia, 2001.

CHARBENEAU, Randall J.; WEAVER, James W.; LIEN, Bob $\mathrm{K}$. The hydrocarbon spill screening model (HSSM): Volume 2: Theoretical Background and Sources Codes. Oklahoma: U.S Environmental Protection Agency, 1995. 229 p. Disponível em:

https://nepis.epa.gov/Exe/ZyPDF.cgi/2000U060.PDF?Dockey $=2000$ U060.PDF. Acesso em: 09 out. 2020

CORSEUIL, Henry Xavier; SCHNEIDER, Marcio Roberto; GIACHINI, Admir José; SOUZA FILHO, André Moreira de. Avaliação da eficiência de impermeabilização de bacias de contenção em áreas petrolíferas. Florianópolis: Ufsc, 2020. 211 p.

CONSELHO NACIONAL DO MEIO AMBIENTE. Resolução $N^{\circ}$ 420, de 28 de dezembro de 2009. Dispõe sobre critérios e valores orientadores de qualidade do solo quanto à presença de substâncias químicas e estabelece diretrizes para o gerenciamento ambiental de áreas contaminadas por essas substâncias em decorrência de atividades antrópicas. Brasil, 2009.

ELETROBRAS. Relatório Anual e de Sustentabilidade 2014: versão resumida para o terceiro setor A energia da inovação e da eficiência. p. 22, 2014.

EMBRAPA. Manual de métodos e análises de solo. p.212. 
EMBRAPA. Sistema brasileiro de classificação de solos Empresa Brasileira de Pesquisa Agropecuaria. Rio de Janeiro, 2006.

GREEN, W. H.; AMPT, G. A. The theory of air and water through soils Title. Journal Agric. Science, v. 4, p. 1-24, 1911.

KARPOUZAS, Dimitrios G.; RIBARBELLI, Carlo; PASTORI, Marco; CAPRI, Ettore. Landscape risk analysis for pesticides applied to rice paddies. Agronomy For Sustainable Development, v. 26, n. 3, p. 167-177, jul. 2006. Springer Science and Business Media LLC. https://doi.org/10.1051/agro:2006013

LIBARDI, Paulo Leonel. Dinâmica da água no solo. São Paulo: Edusp, 2005.

MACHADO, S. L.; DA SILVA PAES CARDOSO, L.; DE OLIVEIRA, I. B.; DE FARIA MARIZ, D.; KARIMPOUR-FARD, M. Modeling Soil Permeability When Percolated by Different Soil. Transport in Porous Media, v. 111, n. 3, p. 763-793, 2016. https://doi.org/10.1007/s11242-016-0627-9

MERCER, J. W.; COHEN, R. M. A review of immiscible fluids in the subsurface: Properties, models, characterization and remediation. Journal of Contaminant Hydrology, v. 6, n. 2, p. 107-163, $\quad 1990 . \quad$ https://doi.org/10.1016/01697722(90)90043-G

MÓDENES, Aparecido Nivaldo; SANDERSON, Karina; TRIGUEROS, Daniela Estelita Goes; SCHUELTER, Adilson Ricken; ESPINOZA-QUIÑONES, Fernando Rodolfo; NEVES, Camila Vargas; ZANÃO JUNIOR, Luiz Antônio; KROUMOV, Alexander Dimitrov. Insights on the criteria of selection of vegetable and mineral dielectric fluids used in power transformers on the basis of their biodegradability and toxicity assessments. Chemosphere, [S.L.], v. 199, p. 312-319, maio 2018. Elsevier BV. https://doi.org/10.1016/i.chemosphere.2018.02.033

NEWELL, C. J.; ACREE, S. S.; ROSS, R. R.; HULLING, S. G.
NUTTING, P. G. Physical analysis of oil sands. Am. Assoc. Petrol.Geol, v. 14, p. 1337-1349, 1934.

NELSON, Jake R; GRUBESIC, Tony H. Oil spill modeling. Progress In Physical Geography. Earth and Environment, [S.L.], v. 42, n. 1, p. 112-127, 12 dez. 2017. https://doi.org/10.1177/0309133317744737

OLIVEIRA, J. C. S. Contaminação de sedimentos argilosos por combustiveis automotivos: problema de avaliação da permeabilidade. p. 129, 2011

PATRICIO, Muriel Edyth Lumsden Szymanski. Modelagem matemática do transporte de óleo mineral isolante na zona não saturada: estudo de caso em subestações de energia elétrica. 2019. 130 f. Dissertação (Mestrado) - Curso de Engenharia Ambiental, Engenharia Ambiental e Sanitária, Universidade Federal de Santa Catarina, Florianópolis, 2019.

PETROBRAS. Ficha de informações de segurança de produtos químicos - FISPQ, 2015.

TOMASELLA, J.; HODNETT, M. Pedotransfer functions for tropical soils. Developments in Soil Science, v. 30, n. C, p. 415-429, 2004. $\quad$ https://doi.org/10.1016/S01662481(04)30021-8

WADA, L. M. Estudo do comportamento de solos contaminados com óleo de isolamento de transformadores. $\mathrm{p}$. 90, 2011.

WEAVER, James W.; CHARBENEAU, Randall J.; LIEN, Bob K.. A screening model for nonaqueous phase liquid transport in the vadose zone using Green-Ampt and kinematic wave theory. Water Resources Research, [S.L.], v. 30, n. 1, p. 93-105, jan. 1994. American Geophysical Union (AGU). https://doi.org/10.1029/93WR02341 